ISSN: 2386-3919 - e-ISSN: 2386-3927

DOI: https://doi.org/10.14201/et2017351117141

\title{
LA ENSEÑANZA DEL INGLÉS PROFESIONAL Y ACADÉMICO BAJO LAS DIRECTRICES EUROPEAS. EL CONTROL AUTÓNOMO DE LA ADQUISICIÓN DE COMPETENCIAS
}

\section{The teaching of profesional and academic English under European directives. The autonomous control of competencies' acquisition}

Pilar DuRÁn ESCRIBANO* y Joana PIERCE MCMAHON** Universidad Politécnica de Madrid. *Correo-e: pilar.duran@upm.es *Correo-e: j.pierce@upm.es

Recibido: 06/06/2016; Aceptado: 26/09/2016; Publicado: 31/05/2017

Ref. Bibl. PILAR DURÁN ESCRIBANO y JOANA PIERCE MCMAHON. La enseñanza del inglés profesional y académico bajo las directrices europeas. El control autónomo de la adquisición de competencias. Enseñanza \& Teaching, 35, 1-2017, 117-141.

RESUMEN: Tras diez años de inmersión en el proceso de convergencia europea de la educación superior, este artículo analiza las fases de aplicación de las directrices europeas, más concretamente, del Marco Común Europeo de Referencia para las lenguas (MCER) y del Portfolio Europeo de Lenguas (PEL) a la enseñanza-aprendizaje-evaluación del inglés profesional y académico (IPA), con la participación de grupos de alumnos de Ingeniería de la Universidad Politécnica de Madrid (UPM) matriculados en asignaturas de IPA. Si bien dichas directrices se habían implementado en otros contextos con resultados varios, su completa adaptación al ámbito de las ingenierías estaba pendiente. El trabajo comienza destacando la coincidencia en la filosofía subyacente al crédito ECTS con las directrices del MCER y del PEL, centrada en el trabajo del estudiante para conseguir determinadas competencias, y en su aprendizaje autónomo y reflexivo. Continúa con la descripción y los resultados de los proyectos de 
investigación y de innovación educativa encaminados a la mejora de la metodología y la gestión del aprendizaje del IPA, destacando la elaboración y aplicación de los descriptores de competencias lingüísticas adaptados al contexto específico. Mediante el análisis de los resultados de una encuesta a los profesores y alumnos participantes, se concluye con la valoración crítica de la implantación de la normativa europea para la enseñanza de IPA en lo que respecta a la actualización de los objetivos y la innovación en los programas, metodologías y sistema de evaluación, en relación con lo que se venía practicando con anterioridad al MCER. Aunque el uso del PEL en su totalidad resulta impracticable, se reconoce su utilidad en los siguientes aspectos: las listas de descriptores de competencias, adaptadas al propio entorno académico y profesional, y el uso del Dosier resultan de gran valor para el universitario, tanto para el aprendizaje autónomo y reflexivo basado en la adquisición de competencias como para desarrollar su capacidad de autoevaluación, como indican los resultados obtenidos.

Palabras clave: EEES; MCER; PEL; competencias comunicativas; aprendizaje autónomo y reflexivo; autoevaluación; IPA.

SUMMARY: After ten years of immersion in the European Higher Education convergence process, this article deals with the application of European directives to the teaching, learning and assessment of English for Academic and Professional Purposes (EAPP) to different groups of engineering students at Universidad Politécnica de Madrid (UPM) enrolled in EAPP subjects. It focuses on the Common European Framework of Reference for languages (CEFR) and the European Language Portfolio (ELP). Although these language-learning directives had been previously implemented in other contexts, with various results, their total adaptation to engineering education was pending. The paper starts by highlighting the coincidence in the principles underlying the European Credit Transfer System (ECTS), and the CEFR and ELP directives centred on the students' work to attain certain competencies, and on their autonomous and reflective learning. Next, it discusses the research and innovation projects aimed at improving teaching practices and learning skills after the application of European directives to EAPP subjects. The main projects dealt with the development and application of language competence descriptors tailored to the specific context. The paper concludes by showing the advantages of their implementation to the improvement of teaching objectives, programme design, teaching methodology and students' evaluation, with respect to other procedures before the CEFR, based on the results of a survey obtained from the teachers and students participating in the study. Although the complete use of the ELP is not feasible, it may be considered as a useful instrument. Both the lists of adapted reference level competence descriptors and the Dossier have proven to be very valuable for the university students' autonomous and reflective learning with the acquisition of competences in mind, and for their self-assessment abilities, according to the results obtained.

Key words: EAHE; CEFR; ELP; communicative competencies; autonomous and reflective learning; self-evaluation; EAPP. 


\section{INTRODUCCIÓN: EL MARCO EUROPEO EN LA ENSEÑANZA DE LENGUAS}

El objetivo principal del Espacio Europeo de Educación Superior (EEes) es la equiparación de los estudios universitarios en todos los Estados de la Unión mediante el sistema europeo de transferencia de créditos (ECTS), que favorece una estructura común en los Grados y Másteres y un control de calidad de las titulaciones. Entre los acuerdos de Bolonia (CEMEs, 1999) destacan la internacionalización de la educación y la movilidad de los estudiantes, para los cuales el conocimiento de lenguas es fundamental, sobre todo del inglés como lingua franca. En este artículo nos centraremos en las fases de la aplicación de las directrices europeas a la enseñanza del inglés profesional y académico (IPA) en el contexto de la ciencia y las ingenierías.

La División de Lenguas Modernas del Consejo de Europa elaboró unas directrices en materia lingüística, recogidas en el Marco Común Europeo de Referencia para las Lenguas (MCER) (Consejo de Europa, 2001), que permiten la equiparación de los niveles de competencia comunicativa de los ciudadanos de la CE en 2L. También recomendó el uso del Portfolio Europeo de Lenguas (PEL) (Consejo de Europa, 1997) para fomentar el plurilingüismo y la gestión del aprendizaje a lo largo de la vida, y así facilitar la movilidad estudiantil y profesional entre los ciudadanos europeos.

La Declaración de Bolonia establece el sistema de créditos ECTs, basado en la carga de trabajo que el estudiante necesita para conseguir unos resultados del aprendizaje claramente establecidos. Se definen como "conjuntos de competencias que expresan lo que el estudiante sabrá, comprenderá o será capaz de hacer tras completar con éxito un proceso de aprendizaje corto o largo" (M. ${ }^{\circ}$ de Educación, 2009: 3). Destacamos aquí el enfoque activo, centrado en el estudiante, al aplicar los créditos ECTS a la enseñanza del IPA.

Por otro lado, el enfoque de las directrices del MCER es pragmático y funcional, ya que contempla el lenguaje en uso para el contexto específico en el que el ciudadano lo necesite: estudio, trabajo, investigación y/o intercambio comercial y cultural. Busca adaptarse a cualquier situación y momento del aprendizaje, respetando las características de cada persona. El MCER «describe de forma integradora lo que tienen que aprender a hacer los estudiantes de lenguas con el fin de utilizar una lengua para comunicarse, así como los conocimientos y destrezas que tienen que desarrollar para poder actuar de manera eficaz» (Consejo de Europa, 2001: 1). Coincide con el enfoque activo, centrado en el alumno, recomendado por Bolonia, concretado en el aprendizaje de lenguas autónomo y reflexivo y el desarrollo de la capacidad de gestionar el aprendizaje a lo largo de la vida; es decir, de programar y autoevaluar el propio progreso. El MCER define seis niveles de dominio de la lengua, que permiten situar el progreso de aprendizaje en cada fase: A1, A2, B1, B2, C1 y C2.

Finalmente, el PEL es el instrumento diseñado por la División de Política Lingüística de la UE para ayudar a implementar las directrices del MCER. Es el CERF's companion piece (Little, 2009: 1), un documento abierto, dividido en tres partes: 
- el pasaporte lingüístico (language passport), que contiene el registro personal de los certificados y experiencias lingüísticas del titular; existe el Europass, accesible electrónicamente y conectado a una central de datos,

- la biografía lingüística (language biography), que ayuda al titular a reflexionar sobre sus competencias comunicativas y estrategias de aprendizaje en diferentes idiomas, y a autoevaluarse mediante el uso de los descriptores de competencias, registrando lo que sabe, comprende y es capaz de hacer, $\mathrm{y}$

- el dosier, que guarda un reflejo de los trabajos del estudiante, cartas, premios y otros documentos de interés lingüístico e intercultural.

Las directrices del PEL (Consejo de Europa, 1997; Schneider y Lenz, 2000) resaltan su doble función: motivar, orientar y apoyar el proceso de adquisición de 2L (función pedagógica), y registrar los distintos niveles homologados alcanzados (función informativa). La validez internacional de la función informativa del PEL radica en su estricta fundamentación sobre los niveles de referencia del MCER. Además, el PEL ${ }^{1}$ fomenta el autoaprendizaje y su autoevaluación, que pueden combinarse con el seguimiento por parte del profesor y la evaluación oficial requerida por las autoridades competentes (Kohonen y Westhoff, 2001). De esta manera, el alumno se motiva al ser el protagonista de su proceso de aprendizaje, contrastando sus logros con los niveles de referencia del MCER.

Nuestro interés en estos años ha sido dar respuesta a las exigencias de adaptación de la metodología de la enseñanza de 2L al EEES, siguiendo las directrices de Bolonia. Según Rivas y Gorgojo (2010: 47), centrarse en los medios que favorecen el acto didáctico y el aprendizaje autónomo es "la piedra angular de esta reforma». Por lo tanto, una tarea importante del profesor será suscitar en el alumno el deseo de aprender y de adquirir unas competencias conocidas y aceptadas con claridad (Rodicio García, 2011: 28). Para ello, tanto el MCER como el PEL son medios de apoyo útiles. Esta tarea de innovación educativa implica una constante relación entre la investigación y la docencia y ha requerido del trabajo en equipo de los profesores de las distintas Escuelas de la UPM que integramos el Grupo de Investigación DISCYT $^{2}$. Dicha relación queda recogida en el artículo, tanto por la referencia a los proyectos competitivos financiados con fondos públicos, como por las publicaciones que exponen los resultados.

1. Existen más de ciento diez modelos de PEL acreditados para primaria, secundaria, formación profesional y la universidad; entre ellos, cinco españoles para cada uno de los niveles citados: cuatro elaborados por el MEC y el ACPEL Portfolio de nivel universitario del que trataremos en este artículo.

2. El grupo de investigación DISCYT de la UPM Estudios cognitivos y sociopragmáticos del discurso científico y técnico lo constituyen siete profesores del Departamento de Lingüística Aplicada de la UPM de las Escuelas de Arquitectura, Aeronáuticos, Agrónomos, Caminos, Minas, Navales, Telecomunicación, y Educación Física y Deportiva. 


\section{El PUNTO DE PARTIDA PARA LA ADAPTACIÓN AL MCER}

La adaptación de las asignaturas de IPA al EeEs conlleva una acción innovadora tanto en el plano metodológico, como en la adecuación de las directrices europeas generales al contexto específico del alumnado de ingeniería y arquitectura. La renovación que plantea el MCER está vinculada al contexto donde ocurre la comunicación; por lo tanto, analizaremos el contexto institucional y revisaremos los niveles de referencia del Marco adaptándolos a las necesidades de aprendizaje de los alumnos (Durán y Roldán, 2007).

\subsection{El contexto institucional}

Para delimitar el contexto, nos valimos de los componentes que influyen en el acto de comunicación establecidos por Hymes (en Renkema, 1993: 44), aplicándolos al ámbito académico del alumnado (Figura 1).

FigURA 1

Componentes de la comunicación

\begin{tabular}{|c|c|}
\hline $\begin{array}{c}\text { COMPONENTES DE } \\
\text { LA COMUNICACIÓN }\end{array}$ & APLICACIÓN A LA INSTITUCIÓN ACADÉMICA UNIVERSITARIA \\
\hline Situación & Formal, académica, profesional \\
\hline Participantes & $\begin{array}{c}\text { Miembros de la comunidad científica (experto a } \\
\text { experto) y receptores interesados (divulgación) }\end{array}$ \\
\hline Finalidad & Objetivo último: la transmisión del conocimiento \\
\hline Registro & Estilo académico, tono formal \\
\hline Canales de & $\begin{array}{c}\text { Clases, conferencias, revistas especializadas y de } \\
\text { divulgación, comunicaciones en congresos, reuniones } \\
\text { técnicas, tecnologías de la información }\end{array}$ \\
\hline Nomunicación & $\begin{array}{c}\text { Establecidas por la amplia comunidad académica, } \\
\text { y por los medios de difusión del conocimiento }\end{array}$ \\
\hline $\begin{array}{c}\text { Géneros (orales } \\
\text { y escritos) }\end{array}$ & $\begin{array}{c}\text { Conferencias; informes técnicos: oral o escrito; artículo } \\
\text { de investigación; abstract; ensayos de divulgación; } \\
\text { instrucciones; medios electrónicos, etc. }\end{array}$ \\
\hline
\end{tabular}

\subsection{Los niveles de referencia del MCER}

Centrándonos en el nivel de inglés requerido para cursar los Grados, constatamos que, aunque cada universidad -incluso dentro de la misma Comunidad Autónoma- tiene sus propias normas, todas ellas sitúan el nivel de exigencia entre B1 y B2, pero no todas ofrecen asignaturas obligatorias ni optativas para conseguirlo (Halbach, Lázaro Lafuente y Pérez Guerra, 2013). La upM exige el nivel B2 y 
ha introducido una asignatura obligatoria de 6 créditos ECTs en todos sus Grados. No obstante, como actualmente el nivel de partida anterior a la universidad es B1 (Prueba de acceso a la Universidad), nuestro trabajo ha hecho hincapié en el desarrollo de los descriptores de destrezas lingüísticas señaladas en el MCER, correspondientes a los niveles B1, B2 y C1, sin olvidar el resto. Esta amplitud ha propiciado que dicho Portfolio pueda servir de referencia para la enseñanza del inglés en otros contextos universitarios semejantes (Bocanegra, 2016: 573).

A pesar de que el MCER puede considerarse como «el documento más influyente y controvertido en el campo de la enseñanza de 2L del siglo XXI» (Figueras 2012: 477), las escalas globales que establece no resultan del todo precisas. Un estudio reciente de Díez-Bedmar (2012) sobre su uso para evaluar las redacciones en inglés de la prueba de acceso a la universidad señala que una de las dificultades más importantes para calificar dichas redacciones radica en que los criterios del MCER son holísticos y cualitativos y, a veces, las marcas diferenciadoras de niveles no son del todo precisas; esto produce una baja relación de fiabilidad en las notas adjudicadas por los distintos evaluadores. Esta misma afirmación, que se ha venido haciendo en otros contextos y niveles educativos (Alderson, 2007; Keddle, 2004; Fulcher, 2004 y 2010; Kuiken, Vedder y Gilabert, 2010), nos llevó a elaborar unos descriptores de competencias más detallados y precisos que las escalas globales y los del MCER, y específicos para el contexto cultural de nuestros alumnos. Los nuevos descriptores de competencias fueron analizados en estudios piloto y baremados según los criterios del MCER, como veremos más adelante

\subsection{El nivel de inglés del alumnado}

En el ámbito académico y profesional, la competencia comunicativa del hablante está vinculada a su competencia lingüística, ya que el lenguaje académico debe ser preciso y correcto (Durán y Rubio, 2015). En el caso de nuestros alumnos de inglés, el nivel de competencia lingüística lo obtuvimos mediante la aplicación del Oxford Placement Test (OPT), una prueba estandarizada y calibrada respecto al MCER. La Figura 2 refleja los datos del porcentaje de alumnos de cada nivel MCER al comienzo de las asignaturas de IPA, en tres cursos académicos distanciados por cuatro años, desde 2005 a 2015. El número de alumnos participantes ha oscilado entre 190 el primer quinquenio, 142 el segundo y 137 el tercero, suficientes para una muestra del nivel del alumnado. 
LA ENSEÑANZA DEL INGLÉS PROFESIONAL Y ACADÉMICO BAJO LAS DIRECTRICES EUROPEAS.

EL CONTROL AUTÓNOMO DE LA ADQUISICIÓN DE COMPETENCIAS

\section{FIGURA 2}

Porcentaje de alumnos según su nivel MCR inicial en tres años académicos

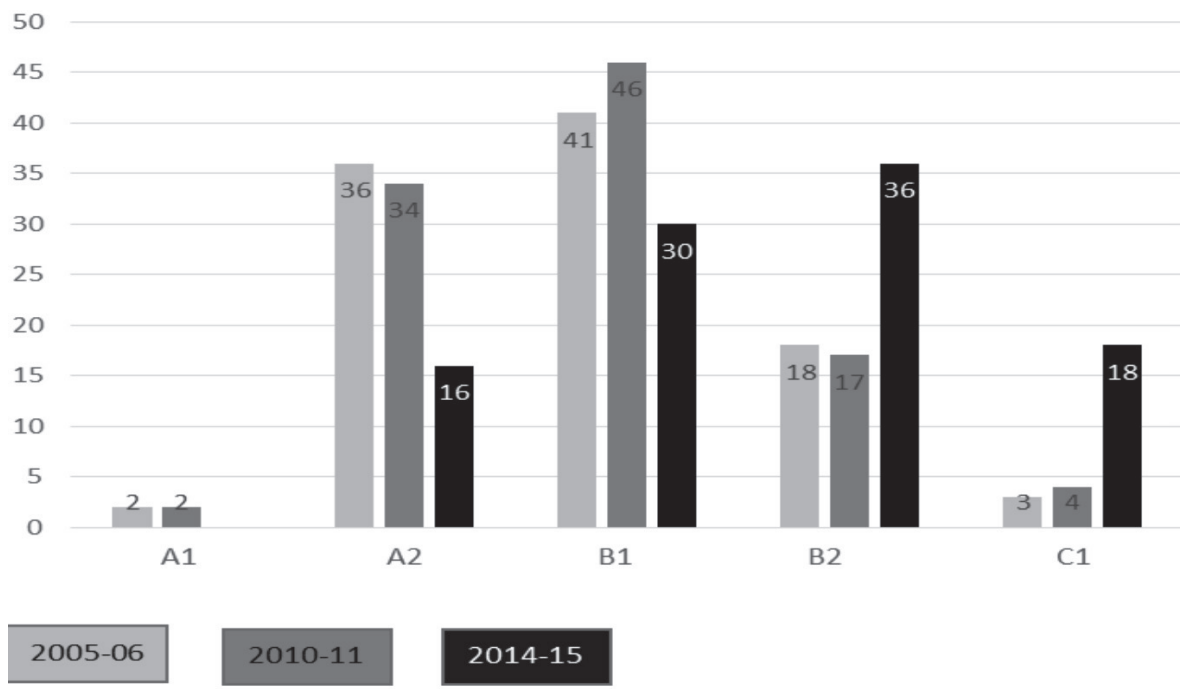

Podemos ver que el nivel inicial de los alumnos de inglés, entre $2 .^{\circ}$ y $4 .^{\circ}$ de carrera, ha ido mejorando en los últimos diez años, aunque observamos cierta dispersión basada en la diversidad de procedencia del alumnado de la UPM (Fig. 2).

- El nivel B1 asignado a las pruebas de idioma en Selectividad, o superior, lo tenían el 62\% de los alumnos en 2005-06, el 64\% en 2010-11 y el 84\% en 2013, al comienzo de las asignaturas obligatorias de inglés.

- El nivel B1, objetivo de las enseñanzas medias, se superó por parte del 21\% en 2005-06, 21\% en 2010-11 y 54\% en 2014-15, según el porcentaje de los que obtuvieron entre B2 y C1 en la prueba de entrada.

- El nivel A1 es prácticamente inexistente y en el A2 se nota un apreciable y progresivo descenso.

Esto parece indicar que los alumnos de enseñanzas medias españoles no sólo reciben una formación cada vez mejor en inglés, sino que son cada vez más conscientes de su necesidad y se preparan por su cuenta para alcanzar el B2 requerido por la mayoría de universidades. Seguramente, el aumento de centros bilingües ha contribuido a ello favorablemente.

También destacamos el hecho de que en la UPM el plan Bolonia se implantó en 2009-10, con la exigencia de que el alumnado demostrara el nivel B2 antes de matricularse en la asignatura obligatoria de «Inglés para la Comunicación Profesio-

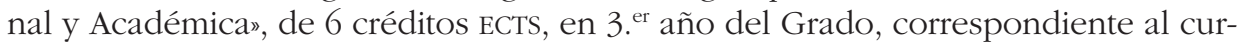
so 2011-12. En cuanto a los datos de los tres grupos que comparamos en la Figura 
PILAR DURÁN ESCRIBANO Y JOANA PIERCE MCMAHON

LA ENSEÑANZA DEL INGLÉS PROFESIONAL Y ACADÉMICO BAJO LAS DIRECTRICES EUROPEAS.

EL CONTROL AUTÓNOMO DE LA ADQUISICIÓN DE COMPETENCIAS

2, solo el último se ve afectado por las exigencias del plan Bolonia; no obstante, el OPT se realizó antes de que los alumnos recibieran autorización para matricularse. A aquellos estudiantes que no alcanzaron el nivel B2 se les ofreció la posibilidad de hacer un curso de nivelación en las propias Escuelas de la UPM.

\section{El PEL ACADÉmiCo y Profesional: ACPEL PORTFOlIO}

\subsection{Fases de desarrollo}

Los profesores del GI DISCYT nos embarcamos, entre 2005 y 2009, en la elaboración de un PEL específico para entornos universitarios científico-técnicos. El denominado ACPEL Portfolio ${ }^{3}$ (Durán et al., 2009) fue acreditado por el Consejo de Europa en 2009. Para ello examinamos otros ejemplares de PEL acreditados, centrándonos en los más próximos a nuestra situación cultural. Destacan el de la Subdirección General de Programas Europeos del Ministerio de Educación y Cultura, modelo para Adultos 16+, N. ${ }^{\circ}$ 59.2004; el "Swiss National Science Foundation Project», modelo N. ${ }^{\circ} 35.202$ http://culture2.coe.int/portfolio/inc.asp y «UK model for adults (with a particular, focus on the learner of languages for vocational purposes)», No 9.2001 www.cilt.org.uk/qualifications/elp/adultelp.

Para su elaboración seguimos los siguientes pasos:

1. Conocer las competencias y estrategias comunicativas de lectura, escritura, y comprensión y expresión oral propias del contexto científico-técnico de la UPM.

2. Estudiar los géneros, tipos de texto y situaciones meta de uso de la lengua que los estudiantes necesitan, y analizar su contenido lingüístico para la correcta formulación de los objetivos competenciales.

3. Detallar las competencias comunicativas siguiendo las pautas del MCER, aplicadas al contexto universitario, que incluyen:

- Competencias lingüísticas y metalingüísticas: léxica, gramatical, semántica, fonológica, ortográfica y ortoépica.

- Competencias sociolingüísticas: marcadores discursivos de las relaciones sociales, fórmulas de cortesía, niveles de formalidad del registro y otros aspectos culturales.

- Competencias pragmáticas: discursiva, funcional y organizativa del texto (Swales, 1990; Bhatia, 1993; Johns, 2002).

3. El proyecto de investigación para la elaboración del Portfolio Europeo de Lenguas Académico y Profesional (ACPEL Portfolio), financiado por la UPM y la Comunidad de Madrid en 2005-06 (PC05/11129. IV PRICYT), es una versión bilingüe del PEL en español e inglés, para educación superior, con fines académicos y profesionales. El ELP Validation Committee lo acreditó con el n. ${ }^{\circ}$ 98.2009, www. coe.int/portfolio (está disponible en info@mairea-libros.com). Recibió el premio a los mejores materiales para la enseñanza de lenguas del Ministerio de Administraciones Públicas en 2010. 
LA ENSEÑANZA DEL INGLÉS PROFESIONAL Y ACADÉMICO BAJO LAS DIRECTRICES EUROPEAS.

EL CONTROL AUTÓNOMO DE LA ADQUISICIÓN DE COMPETENCIAS

4. Elaborar unas listas de descriptores de competencias basados en el MCER y adecuadas a sus seis niveles, con espacios para la autoevaluación y la evaluación por parte del profesor, pero que abarcaran las destrezas y estrategias comunicativas específicas del ámbito propio. Se integraron en un nuevo "Banco de descriptores de destrezas comunicativas» del que se pueden extraer los más adecuados a las necesidades del alumnado en distintos momentos.

5. Elaborar las plantillas para incluir en el Dosier con el fin de facilitar al estudiante cómo ordenar los documentos ilustrativos de lo que es capaz de hacer en una o más 2L, el cv, etc., para mostrarlos oportunamente.

6. Preparar toda la documentación para conseguir la acreditación del ACPEL Portfolio por parte del Comité de Validación del PEL del Consejo de Europa.

\subsection{La biografía lingüistica y el banco de descriptores}

Antes de la edición final del "Banco de Descriptores de Destrezas Comunicativas Profesional y Académico» (Durán et al., 2009)4, se consultaron los ya existentes: Council of Europe 2001; Forster Vosicki, 2002; Lenz y Schneider, 2004 y North, 2007. También se comprobó que todos los descriptores estuvieran redactados con claridad, resultaran relevantes en relación con las necesidades de los alumnos y que existiera una coincidencia estadísticamente aceptable del logro de los objetivos de un nivel con el nivel conseguido por cada alumno. Para constatar su aceptabilidad estadística se utilizaron pruebas externas calibradas con los niveles del MCER, Como el DIALANG, los Cambridge Certificates y el OPT. Los estudios piloto conducentes a la calibración de dichos descriptores han sido publicados por Durán y Pierce, 2007; Pierce y Durán, 2008; Pierce y Robisco 2009; y Pierce y Durán, 2012, 2014.

El nuevo "Banco de Descriptores» (Durán et al., 2009) abarca los seis niveles del MCER agrupados por destrezas: escuchar (46), leer (51), conversar (34), hablar (60), escribir (93), trabajar con textos (43) y competencia comunicativa lingüística (28). En total son 355 descriptores de competencias que sirven de apoyo para la Biografía lingüística de los usuarios del ACPEL Portfolio, y para la programación de asignaturas.

Ejemplos de descriptores ya adaptados y calibrados por nivel, que hacen referencia al lenguaje académico hablado, son los siguientes:

4. El "Banco de Descriptores de Destrezas Comunicativas Profesional y Académico» se publicó conjuntamente con el ACPEL Portfolio (Durán et al., 2009) (disponible en info@mairea-libros.com). 
PILAR DURÁN ESCRIBANO Y JOANA PIERCE MCMAHON

LA ENSEÑANZA DEL INGLÉS PROFESIONAL Y ACADÉMICO BAJO LAS DIRECTRICES EUROPEAS.

EL CONTROL AUTÓNOMO DE LA ADQUISICIÓN DE COMPETENCIAS

\begin{tabular}{|c|c|c|c|c|}
\hline & & 1 & 2 & 3 \\
\hline B1 & $\begin{array}{l}\text { I can understand simple technical information, such as operating } \\
\text { instructions for everyday equipment.- Soy capaz de entender } \\
\text { información técnica sencilla, como las instrucciones de } \\
\text { funcionamiento del equipamiento técnico de uso babitual. }\end{array}$ & & & \\
\hline B2 & $\begin{array}{l}\text { I can follow the essentials of lectures, talks and reports and other } \\
\text { forms of academic or professional presentations in my field. - Soy } \\
\text { capaz de identificar las ideas principales en una clase, en seminarios } \\
\text { e informes o en otras manifestaciones académico-profesionales dentro } \\
\text { de mi campo. }\end{array}$ & & & \\
\hline C1 & $\begin{array}{l}\text { I can follow extended speech on complex topics of academic or } \\
\text { vocational relevance, though I may need to confirm occasional } \\
\text { details, especially if the accent is unfamiliar.- Soy capaz de seguir } \\
\text { comunicaciones orales sobre temas acordes con mis intereses } \\
\text { académicos o profesionales, aunque con apoyo ocasional, sobre todo si } \\
\text { el acento no me resulta familiar. }\end{array}$ & & & \\
\hline
\end{tabular}

\subsection{Los descriptores y el diseño curricular}

Los descriptores, basados en lo que el estudiante necesita saber para desenvolverse en situaciones concretas (target needs), parten del contexto académico y profesional del alumnado descrito en 2.1, para llegar a determinar las metas de aprendizaje (Kohonen y Westhoff, 2001). Además, para apoyar la programación de asignaturas y ayudar a conseguir esas metas, un buen diseño curricular ha de tener en cuenta las necesidades de aprendizaje (learning needs) de los estudiantes, que se basan en el nivel real de competencias lingüísticas del alumnado (Bocanegra, 2016: 563). Esta manera de diseñar el programa contrasta con anteriores posturas en las que la programación y evaluación de una asignatura se basaba casi exclusivamente en la adquisición de los conocimientos del programa oficial correspondiente. Al hacer hincapié en lo que el estudiante será capaz de hacer ("soy capaz de...») se tienen en cuenta tanto las metas de aprendizaje como las necesidades específicas del grupo.

Ejemplos de descriptores utilizados en la programación de asignaturas para alcanzar las competencias lectoras y de escritura adecuadas son: 
PILAR DURÁN ESCRIBANO Y JOANA PIERCE MCMAHON

LA ENSEÑANZA DEL INGLÉS PROFESIONAL Y ACADÉMICO BAJO LAS DIRECTRICES EUROPEAS.

EL CONTROL AUTÓNOMO DE LA ADQUISICIÓN DE COMPETENCIAS

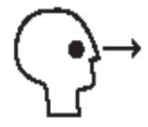

Reading / Leer

B2 I can understand a fairly wide range of specific and more complex texts, with the occasional use of a dictionary, if I can reread difficult sections.- Soy capaz de entender una gama bastante amplia de textos específicos y complejos, con ayuda ocasional del diccionario, si puedo releer las secciones más difíciles.

B2 I can adapt my style and speed of reading to different texts and purposes and consult appropriate reference sources selectively.- Soy capaz de adaptar el estilo y la velocidad lectora a distintos textos y objetivos, y consultar selectivamente las fuentes de referencia adecuadas.

C1 I can use a variety of strategies to understand a complex text, including word building, grammatical and other contextual clues.Soy capaz de usar diversas estrategias para comprender un texto complejo, como por ejemplo la construcción de palabras y otras pistas gramaticales y contextuales.

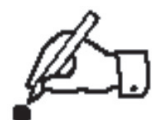

Writing / Escribir

B1 I can write simple connected texts on familiar topics marking relationship between ideas, with reasonable grammatical correctness.Soy capaz de escribir textos sencillos sobre temas conocidos, con cohesión y relacionando las ideas con bastante corrección gramatical.

B2 I can write short essays about academic and professional topics using the special language appropriate for a theme, with occasional errors. - Soy capaz de escribir ensayos cortos sobre temas académicos y profesionales utilizando el vocabulario específico del tema, con errores ocasionales.

B2 I can write a clear, correct and properly sequenced cover letter showing evidence of my motivations, academic preparation and professional experience, making reference to my CV.- Soy capaz de escribir una carta de presentación clara, correcta y bien organizada mostrando mis motivaciones, preparación académica y experiencia profesional.

\subsection{Los descriptores y la autoevaluación}

Antes de dar por válida la función autoevaluadora del ACPEL Portfolio, realizamos un estudio piloto en 2007-08 con alumnos de Ingeniería Técnica de Minas e 
Ingeniería de Minas, que eran cien. Nos preguntamos si los alumnos serían capaces de autoevaluarse de modo válido y fiable, y si podrían detectar sus puntos débiles para mejorarlos y evaluar sus progresos. De los 100 matriculados, solo se contabilizaron los datos de los 49 alumnos que cumplieron los requisitos establecidos.

Preparamos al grupo para seleccionar los descriptores para la autoevaluación de las listas del Banco de Descriptores que les habíamos proporcionado, de la siguiente manera:

a) Determinación conjunta de profesores y alumnos de los objetivos de su asignatura y las destrezas que ellos deberían adquirir, así como de su situación personal respecto a los objetivos y metas de aprendizaje, por medio de las nuevas listas con espacios para la autoevaluación.

b) Apoyo a los alumnos para juzgar su nivel de competencia proporcionándoles criterios concretos y detallados que indicaran el nivel de sus actuaciones en las distintas destrezas (A2, B1, B2, etc.); por ejemplo, mediante escritos ya calificados, como informes técnicos, cartas y descripciones de mapas y otras figuras.

Para decidir el nivel de cada participante, una vez que cada uno había completado las listas de descriptores en las destrezas de lectura y escritura, se estableció el porcentaje mínimo de respuestas afirmativas de cada nivel en el 80\% del total de ambas destrezas. Así, si un alumno marcaba como conseguidos los objetivos de lectura y redacción del nivel B1, en un $80 \%$ o más, se le asignaba dicho nivel. Pero si además marcaba como ya alcanzados el 80\% de los descriptores de ambas destrezas de nivel B2, se le asignaba el nivel superior B2, y así sucesivamente.

Se aplicó los alumnos el OPT al comienzo de la asignatura y se compararon los resultados con los de su autoevaluación. Con estos datos, se obtuvo el número de alumnos que se sobrevaloraban, los que se minusvaloraban y los que coincidían respecto a su nivel MCER del OPT. Los resultados del estudio piloto quedan recogidos en la Figura 3, donde podemos ver que no existen discrepancias significativas entre los niveles obtenidos con el OPT y los resultados de la autoevaluación de las competencias lectoras y de escritura. La mayoría se sitúa entre A2 y B1, pero llaman la atención un 15\% que se autoevalúa como A1 cuando el OPT sitúa en este nivel solo al 2\%; y al contrario, en el otro extremo, los que se sobrevaloran con el nivel B2 y C1. 
PILAR DURÁN ESCRIBANO Y JOANA PIERCE MCMAHON

LA ENSEÑANZA DEL INGLÉS PROFESIONAL Y ACADÉMICO BAJO LAS DIRECTRICES EUROPEAS

EL CONTROL AUTÓNOMO DE LA ADQUISICIÓN DE COMPETENCIAS

\section{FIGURA 3}

Comparativa de porcentajes de puntuaciones en opTest y autoevaluación

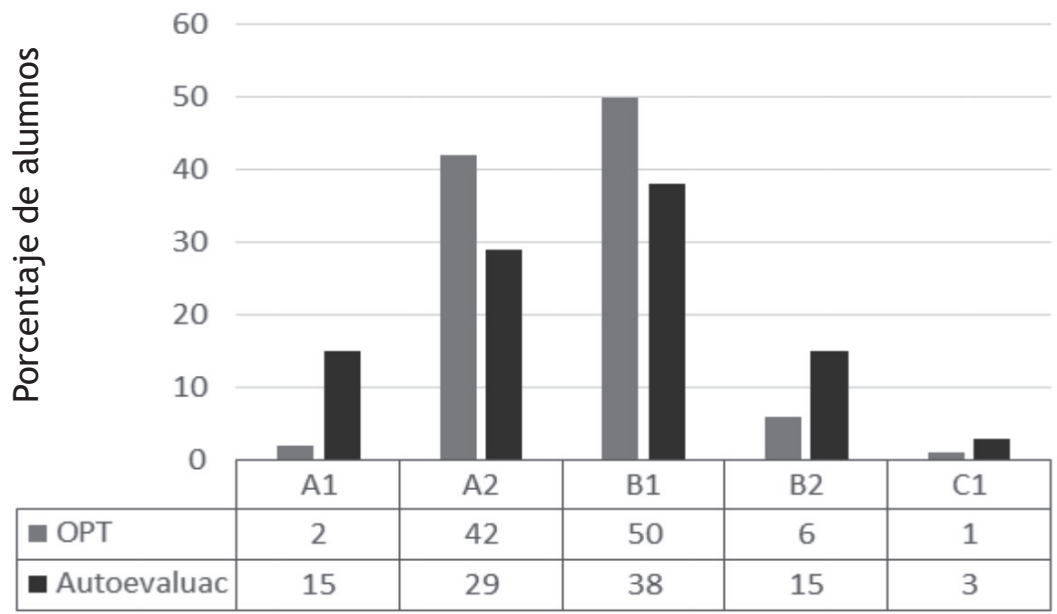

OPT

Autoevaluación

El nivel de significación estadística de los datos se halló utilizando el test no paramétrico de "Wilcoxon Matched Pairs Signed Ranks» (Tabla 1). Se compararon los datos del OPT con la autoevaluación, resultando 10 casos de minusvaloración y 16 de sobrevaloración, que en ningún caso suponían más un nivel de discrepancia, y 23 acuerdos. El test de Wilcoxon, con el nivel de confianza del 5\%, mostró que los resultados de la autoevaluación no eran fruto del azar. Esto parece indicar que los alumnos de esta muestra pueden autoevaluarse en las condiciones anteriormente descritas y que, siguiendo los mismos pasos, otros estudiantes de entornos semejantes también podrían hacerlo.

TABLA 1

Resultados del test «Wilcoxon Matched Pairs Signed Ranks»

\begin{tabular}{|c|c|c|c|}
\hline $\begin{array}{c}\text { WilcoXon (NIVEL DE CONFIANZA 5\%) } \\
(\mathrm{P} \leq 0.05)\end{array}$ & AutOEVALUACIÓN & N. $^{\circ}$ DE CASOS & RANGO MEDIO \\
\hline \multirow{2}{*}{$\begin{array}{c}\text { Oxford Placement Test } \\
\text { y autoevaluación }\end{array}$} & minusvaloración & 10 & 13.75 \\
\cline { 2 - 4 } & sobrevaloración & 16 & 13.34 \\
\cline { 2 - 4 } & coincidencias & 23 & \\
\hline
\end{tabular}




\subsection{El Dosier}

En el Dosier, el titular del ACPEL Portfolio puede guardar los trabajos ilustrativos de su competencia en diversas lenguas, con dos finalidades:

a) El dosier progresivo de trabajo, donde las muestras de los trabajos se guardan para constatar el progreso del estudiante, reflexionar sobre sus avances y autoevaluarse y facilitar la evaluación del profesor en una asignatura reglada.

b) El dosier para presentación (para cambiar de centro o país de estudio, para un trabajo), donde los alumnos archivan aquellos trabajos más representativos de lo que son capaces de hacer y los más adecuados al fin perseguido. Así, por ejemplo, le podría interesar destacar su competencia en diversas lenguas -nacionales o europeas-; alguna competencia concreta, como el conocimiento de un lenguaje técnico, o resaltar alguna destreza, como las presentaciones orales mediante un vídeo. Esta documentación acompaña al cV.

En ambos casos, se recomienda que la colección de trabajos y grabaciones se actualice periódicamente, de acuerdo con nuevos intereses.

\section{APlicación Del aCPel Portfolio EN LA UPM}

Ante la implantación del EEEs, el Vicerrectorado de Ordenación Académica de la UPM convocó ayudas para la realización de Proyectos de Innovación Educativa (PIE) el curso 2008-09. Aunque en varias Escuelas ya habíamos utilizado las listas de descriptores para establecer los objetivos de aprendizaje con los alumnos, los profesores del GI DISCYT y del GIE APLAI asumimos conjuntamente la aplicación del ACPEL Portfolio en siete Escuelas de Ingeniería y Arquitectura, en 2008-095. El objetivo era constatar la validez de la aplicación del nuevo enfoque metodológico centrado en las necesidades del alumnado, utilizando el ACPEL Portfolio. Esta experiencia se consideró innovadora en la universidad española y en el ámbito europeo de la ingeniería y la arquitectura, ya que los PEL existentes de carácter vocacional o profesional hacían referencia a niveles de educación no universitaria.

5. Seguimiento de la adaptación de siete asignaturas curriculares de inglés a los niveles y objetivos del Marco de Referencia Europeo en la UPM, iniciada con el Proyecto ACPEL (PC 05/11129), curso 2008-2009. PIE financiado por la Fundación Gral de la UPM. Referencia IE08100459: "Ayudas a la innovación educativa en el marco del proceso de implantación del EEEs y la mejora de la calidad de la enseñanza» vía Grupo de Innovación Educativa APLAI. Participantes: P. Durán-Escribano y J. Pierce (ETsi Minas y Energía), G. Cuadrado y A. Redondo (eTsi Agrónomos), M. ${ }^{a}$ M. Robisco (ETsi Aeronáuticos), A. Roldán (ETSi Caminos) y P. Úbeda (ETS Arquitectura). 
No se realizó de igual manera en todos los centros por la diversidad de asignaturas propia de la autonomía de las distintas escuelas, aunque las pautas fueron las mismas, como vemos a continuación.

\subsection{Apartados del Proyecto}

La ficha establecida por la UPM, que los profesores implicados habíamos de rellenar para cada asignatura, tenía los siguientes apartados:

1) Objetivos cognitivos y competenciales asignados a las materias en las que se realiza la innovación.

2) Metodologías: métodos utilizados hasta la fecha y nuevas metodologías que se van a aplicar. Explicar cómo se trabajaba con anteriores metodologías y cómo se trabajará con las nuevas.

3) Formas de evaluación: los métodos de evaluación utilizados hasta la fecha y los nuevos métodos que se quiere implantar. Explicar cómo se han utilizado y se utilizarán dichos métodos.

4) Sesiones de tutoría individual o grupal.

5) Recursos de apoyo.

6) Estimación del número de horas de carga de trabajo para el alumno, contabilizando horas lectivas, de trabajo personal o en grupo, y las tutorías.

7) Informe final: con las respuestas de los profesores, basadas en los apartados anteriores, y con las de los alumnos según una encuesta final.

\subsection{Asignaturas y escuelas implicadas}

Las asignaturas implicadas en este estudio se detallan en la Tabla 2. Como podemos ver en la columna «tipo", los créditos y la categoría de obligatoria (Ob), optativa (Op) o libre elección (LE) varía en casi todos los casos; también el número de alumnos que, en total, fue de 276. Un asterisco (*) detrás del número significa que la experiencia se realizó solo con un grupo de la asignatura. 
PILAR DURÁN ESCRIBANO Y JOANA PIERCE MCMAHON

LA ENSEÑANZA DEL INGLÉS PROFESIONAL Y ACADÉMICO BAJO LAS DIRECTRICES EUROPEAS.

EL CONTROL AUTÓNOMO DE LA ADQUISICIÓN DE COMPETENCIAS

TABLA 2

Asignaturas y escuelas implicadas

\begin{tabular}{|c|c|c|c|c|}
\hline ASIGNATURA & TiPO & & Titulaciones & N. ${ }^{\circ}$ ALUMNOS \\
\hline $\begin{array}{l}\text { Fundamentos de } \\
\text { Inglés Técnico }\end{array}$ & $\begin{array}{l}\text { LE/Op } \\
4,5 \mathrm{cr} .\end{array}$ & $\begin{array}{c}\text { ETSI } \\
\text { Minas }\end{array}$ & $\begin{array}{c}\text { Ing. Minas/ } \\
\text { Ing. Geólogo/ } \\
\text { Ing. Técnico Minas }\end{array}$ & 72 \\
\hline Inglés Técnico & $\begin{array}{l}\mathrm{Ob} \\
6 \mathrm{cr}\end{array}$ & $\begin{array}{c}\text { ETSI } \\
\text { Minas }\end{array}$ & Ing. Técnico Minas & $24^{*}$ \\
\hline Inglés II & $\begin{array}{l}\mathrm{Ob} \\
9 \mathrm{cr} .\end{array}$ & $\begin{array}{c}\text { ETSI } \\
\text { Caminos }\end{array}$ & $\begin{array}{l}\text { Ing. Caminos, } \\
\text { Canales y Puertos }\end{array}$ & $50^{*}$ \\
\hline $\begin{array}{l}\text { Inglés Académico } \\
\text { y Profesional }\end{array}$ & $\begin{array}{l}\mathrm{LE} \\
3 \mathrm{cr} \text {. }\end{array}$ & $\begin{array}{c}\text { ETSI } \\
\text { Agrónomos }\end{array}$ & Ing. Agrónomo & 20 \\
\hline Traducción Técnica & $\begin{array}{l}\mathrm{LE} \\
4,5 \mathrm{cr} \text {. }\end{array}$ & $\begin{array}{c}\text { ETSI } \\
\text { Agrónomos }\end{array}$ & Ing. Agrónomo & 25 \\
\hline $\begin{array}{l}\text { Comunicaciones } \\
\text { Profesionales }\end{array}$ & $\begin{array}{l}\mathrm{LE} \\
4,5 \mathrm{cr} \text {. }\end{array}$ & $\begin{array}{c}\text { EUIT } \\
\text { Aeronáutica }\end{array}$ & $\begin{array}{l}\text { Ing. Técnico } \\
\text { Aeronáutico }\end{array}$ & 25 \\
\hline $\begin{array}{l}\text { Aplic. Profesionales } \\
\text { en Inglés para Arquit }\end{array}$ & $\begin{array}{l}\mathrm{LE} / \mathrm{Op} \\
4,5 \mathrm{cr} .\end{array}$ & $\begin{array}{c}\text { ETS } \\
\text { Arquitectura }\end{array}$ & Arquitecto & $60 *$ \\
\hline
\end{tabular}

\subsubsection{Objetivos de las asignaturas}

Desglosamos los objetivos de las materias en: a) los comunes a todas ellas y b) los específicos de cada asignatura.

a) Objetivos cognitivos y competenciales comunes. Estos fueron acordados por todas las profesoras al solicitar el PIE y están relacionados con las directrices del MCER y los fines del ACPEL Portfolio:

- Conocer lo que supone el EEEs y el MCER para las lenguas.

- Describir las propias competencias comunicativas en inglés y marcar nuevas metas de aprendizaje utilizando los nuevos descriptores de competencias.

- Desarrollar hábitos de aprendizaje autónomo y reflexivo para 'aprender a aprender' 2L y poder planificar y desarrollar el propio aprendizaje a lo largo de la vida.

- Adquirir habilidades de autoevaluación y de evaluación de otros en relación con los objetivos competenciales referenciados al MCER (ver $3.2,3.3$ y 3.4$)$.

b) Objetivos competenciales específicos de las asignaturas, principalmente los dirigidos a la adquisición de destrezas de comprensión lectora y de expresión escrita en algunas asignaturas, y los centrados en la conversación, expresión oral y presentaciones profesionales, en otras: 
LA ENSEÑANZA DEL INGLÉS PROFESIONAL Y ACADÉMICO BAJO LAS DIRECTRICES EUROPEAS.

EL CONTROL AUTÓNOMO DE LA ADQUISICIÓN DE COMPETENCIAS

- Utilizar técnicas de lectura rápida e intensiva para localizar información y extraer las ideas fundamentales de un texto.

- Desarrollar técnicas de deducción de significado para las palabras nuevas y detectar los 'falsos amigos'.

- Utilizar adecuadamente el diccionario (formatos papel y electrónico) para seleccionar el significado de la palabra según el contexto.

- Utilizar las claves morfológicas y el orden adecuado de las oraciones al redactar y traducir directa e inversamente inglés/español.

- Distinguir los distintos tipos de texto y su estructura.

- Redactar los tipos de escrito acordados al comienzo de cada asignatura con fluidez y coherencia.

- Adquirir estrategias para la planificación, desarrollo, revisión y corrección de textos antes de su presentación, oralmente o por escrito.

- Conocer los elementos que intervienen en un acto de comunicación y valorar la audiencia.

\subsection{Innovación en metodología}

TABLA 3

\begin{tabular}{|l|l|}
\hline \multicolumn{1}{|c|}{ MÉTODOS Y/O ORIENTACIÓN ANTERIORES } & NUEVOS MÉTODOS Y/O NUEVA ORIENTACIÓN \\
\hline $\begin{array}{l}\text { Motivar al alumno para aprobar la } \\
\text { asignatura y completar su carrera }\end{array}$ & $\begin{array}{l}\text { Suscitar el deseo de aprender y adquirir } \\
\text { competencias concretas }\end{array}$ \\
\hline $\begin{array}{l}\text { Lección magistral con apoyo de } \\
\text { Power Point o transparencias, } \\
\text { utilizado por profesor }\end{array}$ & $\begin{array}{l}\text { Uso de Power Point o transparencias, por } \\
\text { el profesor para sus explicaciones y por } \\
\text { alumnos para presentaciones en aula }\end{array}$ \\
\hline $\begin{array}{l}\text { Actividades grupales: tareas sobre un texto } \\
\text { con algún compañero, seguidas de su } \\
\text { corrección en el transcurso de la clase }\end{array}$ & $\begin{array}{l}\text { Actividades grupales: tareas sobre un texto } \\
\text { con algún compañero, seguidas de su } \\
\text { corrección en el transcurso de la clase }\end{array}$ \\
\hline & $\begin{array}{l}\text { Aprendizaje cooperativo: realización de } \\
\text { tareas para fomentar la interdependencia } \\
\text { positiva }\end{array}$ \\
\hline $\begin{array}{l}\text { Prácticas en el aula multimedia. } \\
\text { Realización de ejercicios de ordenador } \\
\text { y audiciones, iguales para todo } \\
\text { el grupo (14-15 prácticas). }\end{array}$ & $\begin{array}{l}\text { Prácticas en el aula multimedia. } \\
\text { Proporcionar un número mayor (20) de } \\
\text { prácticas para atender la diversidad de } \\
\text { niveles del alumno, que elegirá las más } \\
\text { adecuadas a su nivel, con mínimo de } 14\end{array}$ \\
\hline & \begin{tabular}{l} 
Apoyo de las TIC (plataforma moodle) \\
\hline
\end{tabular} \\
\hline $\begin{array}{l}\text { Uso de las listas de descriptores de } \\
\text { competencias para marcar objetivos y } \\
\text { autoevaluarse }\end{array}$ \\
\hline
\end{tabular}




\subsection{Innovación en evaluación}

TABLA 4

\begin{tabular}{|l|l|}
\hline \multicolumn{1}{|c|}{ FORMAS DE EVALUACIÓN (SUMATIVA) } & \multicolumn{1}{c|}{ NUEVAS FORMAS DE EVALUACIÓN } \\
\hline Evaluación continua preferentemente & Evaluación continua preferentemente \\
\hline Pruebas periódicas tipo examen breve & $\begin{array}{l}\text { Pruebas periódicas: calificación de tareas } \\
\text { acordadas }\end{array}$ \\
\hline Prácticas & Prácticas \\
\hline Participación en clase & Participación en clase \\
\hline & $\begin{array}{l}\text { Autoevaluación del propio progreso por } \\
\text { medio de listas de descriptores }\end{array}$ \\
\hline $\begin{array}{l}\text { Examen final si no supera la } \\
\text { evaluación continua }\end{array}$ & $\begin{array}{l}\text { Examen final si no supera la } \\
\text { evaluación continua }\end{array}$ \\
\hline
\end{tabular}

\subsection{Encuesta final a los alumnos}

El PIE finalizaba con una encuesta al alumnado con preguntas comunes a todas las asignaturas y otras más específicas de cada materia, según las siguientes pautas:

Indica entre 4 y 1 cómo calificarías los siguientes elementos en relación con tu aprendizaje en la asignatura ( $4=$ muy alto; $3=$ alto; $2=$ medio; $1=$ bajo):

1. Qué es el EEEs ${ }^{6}$

2. Aprendizaje global, mejora de competencias

3. Uso del idioma inglés (expresión oral)

4. Uso de la red

5. Valoración del programa y de los objetivos específicos

6. Trabajo en aula (grupo grande o subgrupos)

7. Trabajo personal

8. Método de evaluación

9. Asistencia a clase

10. Satisfacción global al cursar la asignatura

6. Esta pregunta se incluye como requisito del Rectorado en la convocatoria de PIE (ver 4.2.1) 


\section{RESUlTADOS DE LA EXPERIENCIA}

\subsection{Valoración de las competencias adquiridas por los alumnos}

Analizadas las fichas cumplimentadas por los profesores (ver 4.1 y 4.2), presentamos los resultados de la encuesta de un total de 96 alumnos de tres grupos, de las titulaciones de Ingeniero de Minas, Ingeniero Geólogo e Ingeniero Técnico de Minas, que cumplieron todos los requisitos del PIE. Los datos corresponden a las asignaturas de "Fundamentos de Inglés Técnico» e "Inglés Técnico» (Tabla 2), y se centran en las competencias de comprensión lectora y de expresión oral y escrita sobre temas relacionados con sus estudios. Otras dos asignaturas trabajaron con el Dosier, y las restantes no aplicaron la encuesta a los alumnos.

FIGURA 4

Valoración (4-1) de las competencias adquiridas por los alumnos

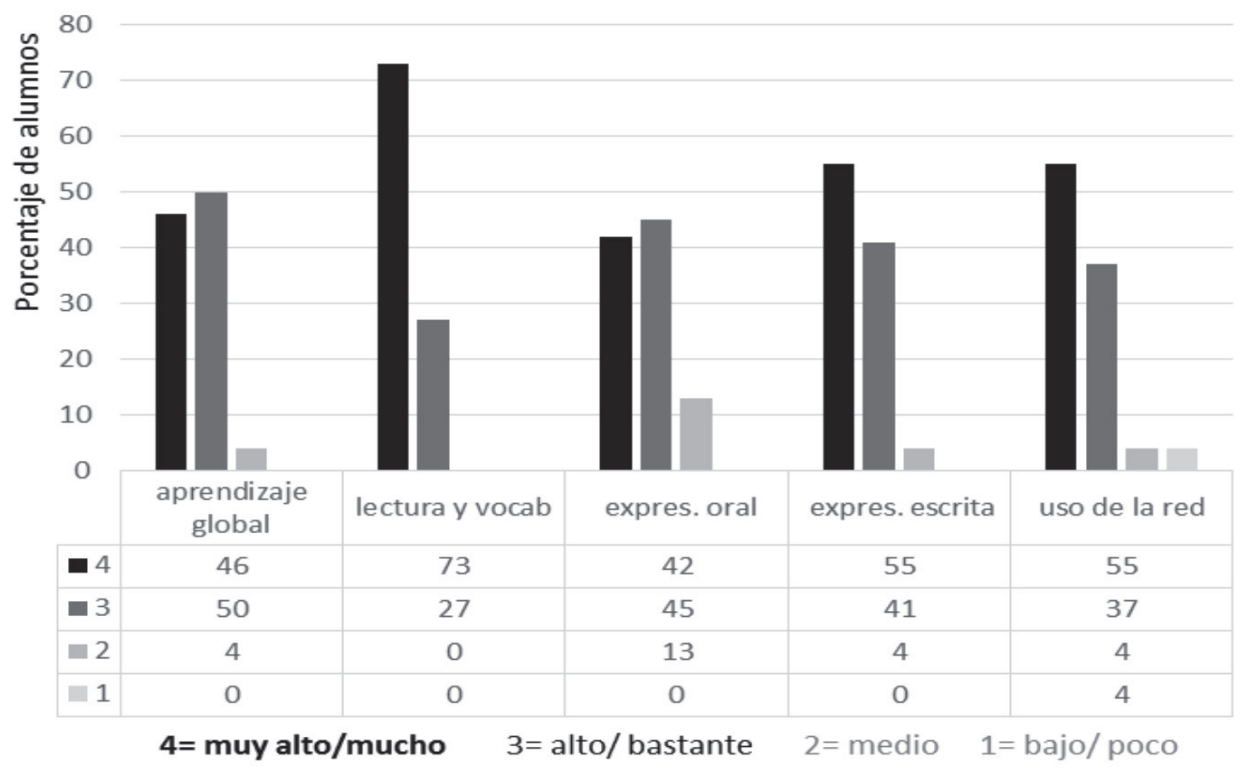

La Figura 4 revela que la gran mayoría de las respuestas oscilan entre 4 (muy alto) y 3 (alto); un alto porcentaje de los 96 alumnos implicados dicen haber mejorado mucho o bastante en las competencias comunicativas meta del aprendizaje. Destaca la valoración positiva en comprensión lectora y vocabulario específico, con un $73 \%$ de alumnos que califican su aprendizaje de "muy alto" y un $27 \%$ alto, con ausencia de valoraciones medias o bajas. Por el contrario, en expresión oral la dispersión de respuestas es mayor: el 42\% dicen haber mejorado mucho, el 45\% bastante y el 13\% señala un nivel medio. Las competencias en expresión escrita y uso de la red 
coinciden bastante; sin embargo, en el uso de la red hay un grupo de alumnos (4\%) que dice haber mejorado poco, quizá porque partían de un buen nivel.

\subsection{Valoración de la gestión del aprendizaje}

FIGURA 5

Valoración (4-1) de la gestión del proceso de aprendizaje

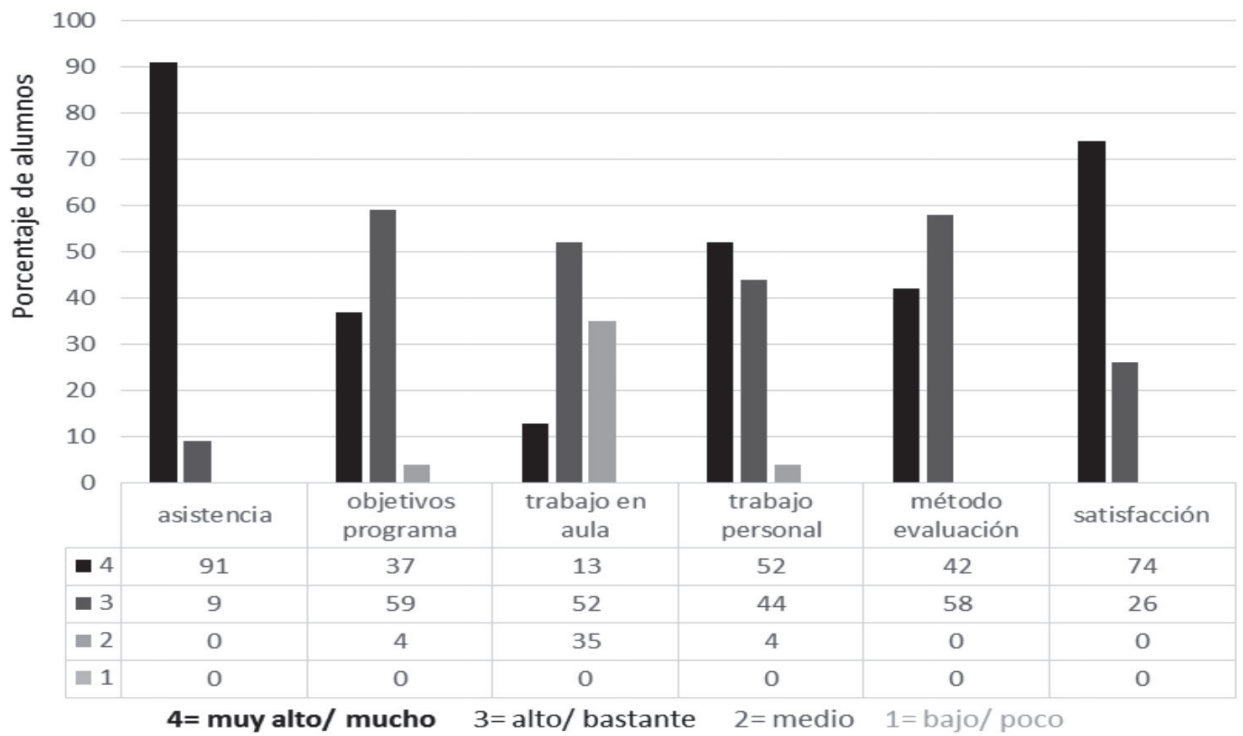

En cuanto a la gestión del aprendizaje, acordada entre alumnos y profesoras de los tres grupos analizados, la valoración de los distintos elementos se sitúa mayoritariamente entre 4 (muy alto) y 3 (alto) (Figura 5). Destacan la muy alta asistencia regular a clase del 91\% de los estudiantes, y que la valoración del trabajo personal se sitúa por encima del trabajo en aula. El acceso tanto programado como libre al aula multimedia para trabajar con audiciones y web-quests, y para realizar ejercicios gramaticales de refuerzo, etc., se valoró como un elemento de aprendizaje con resultados superiores a los conseguidos al trabajar en grupo. Las diferencias de nivel de los alumnos en las distintas destrezas hacen que el trabajo personal sea un recurso imprescindible para la enseñanza de 2L a universitarios (Ortega, 2015; Pierce y Durán, 2009).

Tanto el método de evaluación como el conocimiento de los objetivos del programa reciben una valoración de 3 (alta) por el 58\% y 59\%, y de 4 (muy alta), por el $42 \%$ y $37 \%$ del alumnado, respectivamente. No es de extrañar que, habiendo participado en la determinación de los objetivos del programa y en su evaluación, los alumnos muestren satisfacción con ello. 
LA ENSEÑANZA DEL INGLÉS PROFESIONAL Y ACADÉMICO BAJO LAS DIRECTRICES EUROPEAS.

EL CONTROL AUTÓNOMO DE LA ADQUISICIÓN DE COMPETENCIAS

\subsection{Los objetivos generales comunes}

En lo que se refiere a los objetivos generales del PIE, las respuestas del total de $276^{7}$ estudiantes a las preguntas comunes a todas las asignaturas implicadas son las siguientes:

1) Conocimiento del EeEs y de la finalidad del MCER para las lenguas.

Las respuestas fueron satisfactorias: 28 sujetos, el 10\%, lo valoraron en 4 (muy alto); 176, el 64\%, en 3 (alto); y 72, el 26\%, en 2 (medio).

2) Capacidad para reflexionar y planificar las propias metas de aprendizaje. Las respuestas de los alumnos sobre su capacidad para planificar el aprendizaje fueron: 96 sujetos, el 35\%, lo valoraron en 4 (muy alto); 171, el 62\%, en 3 (alto); y 9, el 3\%, en 2 (medio). Estos datos resultan muy satisfactorios: la gran mayoría valoró su capacidad de aprendizaje autónomo y reflexivo como alto o muy alto después de cursar las asignaturas. Las pautas para ayudarles a reflexionar sobre sus competencias comunicativas, el trabajo personal y la disponibilidad horaria del aula multimedia fueron elementos decisivos para alcanzar estos objetivos.

3) Adquisición de habilidades de autoevaluación y de evaluación de otros en relación con los niveles del MCER.

Este objetivo obtuvo unos resultados muy semejantes al anterior: 101 sujetos, el 37\%, lo valoraron en 4 (muy alto); 166, el 60\%, en 3 (alto); y 9, el 3\%, en 2 (medio). Las respuestas reflejan que las actividades realizadas para favorecer la capacidad del alumnado para autoevaluarse resultaron satisfactorias. Tanto esta capacidad como la de planificar su aprendizaje con metas realistas son fundamentales para apoyar el aprendizaje de lenguas a lo largo de la vida, algo esencial en un mundo globalizado.

4) Finalmente, sobre la satisfacción global con el proceso de aprendizaje, las respuestas fueron claramente positivas: 206 estudiantes, el 75\%, muestran un grado muy alto de satisfacción; 66, el 24\%, un grado alto; y 4, el 1\%, un grado medio.

La aplicación del ACPEL Portfolio en la UPM ha supuesto la adaptación a una metodología centrada en el alumno, para todos los implicados. Los alumnos que en un principio pudieron ofrecer cierta resistencia al cambio pronto valoraron positivamente el sentirse protagonistas de su proceso de aprendizaje.

7. A este bloque de preguntas respondió el total de los alumnos implicados (ver Tabla 2). 


\subsection{El uso del Dosier}

El Dosier, también llamado carpeta de aprendizaje o portfolio en otros ámbitos fuera del lingüístico, se ha utilizado como nueva metodología docente, motivadora, con buenos resultados (Cabero-Almenara et al., 2014; Cayón-Costa y Fuentes-Agustí, 2014). En nuestro caso, se utilizó con éxito para la evaluación en dos escuelas: Arquitectura e Ingenieros Agrónomos, trabajando con los dos tipos de Dosier.

a) El dosier progresivo de trabajo, con las muestras de ejercicios de los alumnos, se utilizó en la ETSI Agrónomos en la asignatura de traducción. Sirvió para que el estudiante constatara su progreso, reflexionara sobre sus avances y se autoevaluara, así como para facilitar la evaluación del profesor.

b) En Arquitectura, donde los alumnos están muy acostumbrados a utilizar Portafolios en diversas asignaturas, el Dosier lingüístico de la modalidad dosier para presentación resultó de gran utilidad. Los alumnos seleccionaron aquellos trabajos y materiales más representativos de lo que eran capaces de hacer y más adecuados al fin perseguido (admisión en otro centro, entrevista de trabajo), destacando su competencia en diversas lenguas, el conocimiento del lenguaje técnico inglés o el diseño gráfico rotulando en inglés (Úbeda-Mansilla y Roldán-Riejos, 2012).

A los alumnos se les animó a que actualizaran periódicamente la colección de trabajos y grabaciones, de acuerdo con sus intereses. Este método de trabajo, además de los encuentros en clase, llevó consigo la realización de, al menos, dos tutorías largas, personalizadas. No podemos ocultar que, en la universidad, con números elevados de alumnos (por encima de 30/aula y varios grupos), el uso del Dosier, que presupone un aumento de tutorías, puede resultar pesado. Aunque la experiencia fue positiva, los profesores implicados consideran que no se puede realizar bien con todos los grupos. Es preciso encontrar un equilibrio al introducir nuevas metodologías docentes, que produzca un mayor rendimiento académico sin sobrecargar al profesor.

\section{CONClusiones}

En este artículo hemos expuesto el proceso de adaptación de la enseñanza-aprendizaje del IPA al EeEs dentro del contexto de la UPM. Actualizar su metodología ha supuesto para el profesorado comprender los cambios planteados por la sociedad globalizada y del conocimiento. La internacionalización de la educación y la movilidad de profesionales exigen un conocimiento alto del inglés y la apertura a distintas culturas. El EEES demanda una educación centrada no solo en lo que el estudiante debe saber, sino en lo que debe ser capaz de hacer y en lo que debe valorar.

Detectada la necesidad de desarrollar las escalas y adaptar los descriptores de competencias lingüísticas del MCER a la universidad, se emprendió un proyecto que 
duró cuatro años hasta conseguir que las competencias lingüísticas requeridas por los estudiantes de ciencias e ingenierías estuvieran bien definidas y se convirtieran en el centro del aprendizaje del IPA. La innovación, por tanto, se ha centrado no solo en los contenidos -enfocados a la comunicación académica y profesional-, sino en su didáctica, con un nuevo enfoque metodológico basado en las necesidades lingüísticas del alumnado y su participación activa en todo el proceso.

El ACPEL Portfolio, como instrumento innovador para el aprendizaje de lenguas, ha resultado útil. Al comentar los resultados de la aplicación del ACPEL Portfolio en siete asignaturas de IPA, hemos descrito los pasos para involucrar a los alumnos, desarrollar su capacidad de aprendizaje autónomo y reflexivo y autoevaluarse. En la evaluación continua, sumativa, se tuvieron en cuenta todos los datos relativos al logro de competencias de cada alumno: los aportados por él mismo y los constatados por las profesoras. Sus respuestas fueron muy favorables respecto al aprendizaje, a su gestión y al método de evaluación.

Hemos observado que trabajar con el ACPEL Portfolio en su totalidad resulta impracticable por la ratio alumnos/profesor y el tiempo que ello conllevaría. Así, en unas asignaturas se ha podido trabajar satisfactoriamente con las listas de descriptores de competencias de la Biografía lingüística y completar el Pasaporte lingüístico, mientras que en otras se ha trabajado con el Dosier con resultados muy satisfactorios, en línea con los hallazgos de Cayón-Costa y Fuentes-Agustí (2014: 60-61). En ambos casos, la implicación del alumnado, su asidua asistencia a clase, la alta valoración del aprendizaje autónomo y su capacidad de autoevaluación dan buena muestra de la eficacia del enfoque comunicativo, activo y personalizado, en la enseñanza de IPA, propugnado por las directrices europeas.

Las "Guías de Aprendizaje», con las que se trabaja en la actualidad, recogen estos principios y hacen referencia a los nuevos descriptores de competencias calibrados respecto al MCER. El uso del Dosier se sigue recomendando, no solo para motivar y evaluar al alumno, sino para prepararle para su entrada en el mundo profesional.

\section{REFERENCIAS BIBLIOGRÁFICAS}

Alderson, J. C. (2007). The CERF and the need for more research. The Modern Language Journal, 91 (4), 659-663.

Bhatia, V. (1993). Analysing Genre: Language Use in Professional Settings. Essex: Longman. Bocanegra-Valle, A. (2016). Needs Analysis for Curriculum Design. En K. Hyland y P. Shaw (Eds.). Handbook of English for Academic Purposes (pp. 562-578). Abingdon, Oxford: Routledge.

Cabero-Almenara, J.; López-Meneses, E. y Jaén-Martínez, A. (2014). Los portafolios educativos virtuales en las aulas universitarias. Instrumentos didácticos para la innovación docente y la calidad de los procesos de enseñanza y aprendizaje. Enseñanza \& Teaching: Revista Interuniversitaria de Didáctica, 31 (1), pp 43-70. 
Cayón-Costa, M. y Fuentes-Agustí, M. (2014). Incidencia de las carpetas de aprendizaje en el rendimiento académico de los estudiantes universitarios. Enseñanza \& Teaching: Revista Interuniversitaria de Didáctica, 32 (1), 59-78.

CEMES (Conferencia Europea de Ministros de Educación Superior) (1999). Declaración de Bolonia. El Espacio Europeo de Educación Superior (1999). Bolonia, Italia.

Consejo de Europa (1997). European Language Portfolio: proposals for development. Strasbourg: Council of Europe.

Consejo de Europa (2001). Common European Framework of Reference for Languages: Learning, teaching, assessment. Cambridge: CuP.

Díez Bedmar, B. (2012). El uso del Marco Común Europeo de Referencia para las Lenguas para evaluar las redacciones en la sección de inglés de la Prueba de Acceso a la Universidad. Revista de Educación, 357, 55-80.

Durán-Escribano, P.; Cuadrado, G.; Pierce, J.; Roldán, A.; Robisco, M. ${ }^{a}$ M.; Santiago, J. y Úbeda, P. (2009). Portfolio Europeo de las Lenguas Académico y Profesional. ACPEL Portfolio y Banco de Descriptores de Destrezas Comunicativas Profesional y Académico. Madrid: Ediciones Endymión.

Durán-Escribano, P. y Pierce, J. (2007). An EAP literacy portfolio for tertiary education students: Academic and professional discourse development within the European framework of reference for languages. En A. Curado, P. Edwards y M. Rico (Eds.). Approaches to Specialised Discourse in Higher Education and Professional Contexts (pp. 182-197). Newcastle, UK: Cambridge Scholars.

Durán-Escribano, P. y Roldán, A. (2007). Desarrollo de un modelo de Pel de Inglés Académico y Profesional para la UPM. En Aprendizaje de Lenguas, uso del Lenguaje y Modelación Cognitiva, XXIV Congreso Internacional de AESLA (pp. 983-989). Madrid, UNED.

Durán-Escribano, P. y Rubio-Moreda, A. L. (2015). Evaluación del uso competente de los rasgos lingüísticos del IPA en relación con los niveles del MCER. Revista de Lingüústica y Lenguas Aplicadas, 10, 21-33.

Figueras, N. (2012). The Impact of the CERF. ELT Journal, 66 (4), 477-485.

Forster Vosicki, B. (2002). European Language Portfolio for Higher Education. Bern, CH: European Language Council.

Fulcher, G. (2004). Are Europe's tests being built on an 'unsafe' framework? Education Guardian. http://www.theguardian.com/education/2004/mar/18/tefl2.

Fulcher, G. (2010). The reification of the Common European Framework of Reference (CEFR) and effect-driven testing. Advances in research on language acquisition and Teaching: Selected Papers, pp. 15-26.

Halbach, A; Lázaro Lafuente, A. y Pérez Guerra, J. (2013). La lengua inglesa en la nueva universidad del EEES. Revista de Educación, 362, 105-132.

Johns, A. M. (2002). Genre in the Classroom. Multiple Perspectives. New Jersey: Lawrence Earlbaum Associates.

Keddle, J. S. (2004). The CEF and the secondary school syllabus. En K. Morrow (Ed.). Insights from the Common European Framework (pp. 43-54). Oxford: OUP.

Kohonen, V. y Westhoff, G. (2001). Enhancing the Pedagogical Aspects of the European Language Portfolio (ELP). Strasbourg: Council of Europe.

Kuiken, F.; Vedder, I. y Gilabert, R. (2010). Communicative Adequacy and Linguistic Complexity in L2 writing. En I. Bartning, M. Martin y A. Vedder (Eds.). Communicative proficiency and linguistic development: intersections between SLA and language testing research (pp. 81-100). Eurosla Monographs, 1. 
LA ENSEÑANZA DEL INGLÉS PROFESIONAL Y ACADÉMICO BAJO LAS DIRECTRICES EUROPEAS.

EL CONTROL AUTÓNOMO DE LA ADQUISICIÓN DE COMPETENCIAS

Lenz, P. y Schneider, G. (2004). A bank of descriptors for self-assessment in European Language Portfolios. Council of Europe; Strasbourg. Recuperado de www.coe.int/portfolio.

Little, D. (2009). The European Language Portfolio: where pedagogy and assessment meet. En $8^{\text {th }}$ International Seminar on the ELP. DGIV EDU LANG 2009: 19.

Ministerio de Educación (2009). Guía del usuario del ECTS. Madrid 6-02-2009.

North, B. (2007). Council of Europe data bank of descriptors: 'CEFR illustrative descriptors, section 2', and 'Expanded set of C1 and C2 descriptors'. Eurocentres. www.coe.int/ portfolio.

Ortega, L. (2015). Syntactic complexity in L2 writing: Progress and expansion. Journal of Second Language Writing, 29, 82-94.

Pierce, J. y Durán, P. (2008). Language Communication Competences in Engineering Education Revisited. Revista de Lingüistica y Lenguas Aplicadas, 3, 59-72.

Pierce, J. y Durán, P. (2009). Information technology: the use of webquests in ESP. En $V I$ Jornadas Internacionales de Innovación Universitaria. Madrid: Universidad Europea de Madrid.

Pierce, J. y Durán, P. (2012). Analysing written production competence descriptors for academic and professional purposes and their calibration to the CERF. IBERICA, 23, 109-130.

Pierce, J. y Durán, P. (2014). Developing and Evaluating Self-Assessment Reading Competence Descriptors for the ELP. Porta Linguarum. Revista Internacional de Didáctica de las Lenguas Extranjeras, 22, 127-146.

Pierce, J. y Robisco, M. ${ }^{a}$ M. (2010). Evaluation of oral production learning outcomes for higher education in Spain. Assessment and Evaluation in Higher Education, 35 (6), 745-758.

Renkema, J. (1993). Discourse Studies. Amsterdam: John Benjamins Publishing Co.

Rivas, M. R. y Gorgojo, M. C. S. (2010). El trabajo en las aulas con perspectiva europea: Medios y recursos para el aprendizaje autónomo. Enseñanza \& Teaching: Revista Interuniversitaria de Didáctica, 28 (2), 45-60.

Rodicio-García, M. L. (2011). La docencia universitaria en el proceso de adaptación a Europa: la percepción de los estudiantes. Enseñanza \& Teaching: Revista Interuniversitaria de Didáctica, 28 (2), 23-43.

Schneider, G. y P. Lenz (2000). European Language Portfolio: Guide for Developers. Fribourgh, CH: University of Fribourgh.

Swales, J. (1990). Genre Analysis. English in Academic and Research Settings. Cambridge: C.U.P.

Úbeda-Mansilla, P. y Roldán-Riejos, A. (2012). Professional Applications in English for Architects: The Interview Guide. En Proceedings of EDULEARN 12 Conference (pp. 4816-4824). Barcelona: EDULERN. 